\title{
Noncommutative Geometry, background independence, and $B-L$ extension of the Standard Model
}

\author{
Fabien Besnard* \\ $E P F$ \\ E-mail: fabien.besnardeepf.fr
}

\begin{abstract}
In these notes we present a new framework, called "algebraic backgrounds", which is a slight modification of Connes' Spectral Triples theory which allows for a transparent representation of diffeomorphisms and spin structure equivalences. In an algebraic background there is no fixed Dirac operator but, instead, a bimodule of noncommutative 1-forms which plays the role of the differential structure of the manifold. The configuration space is the space of Dirac operators which are compatible with this bimodule. They play the role of metrics compatible with the differential structure. We present the cases of manifolds and finite graphs as motivations. The symmetry group of the algebraic background associated to a manifold turns out to be exactly that of the tetradic formulation of General Relativity, but the configuration space contains extra fields, not associated with a metric. However the projection on metric fields is diffeomorphism invariant so that they can be safely removed. The same framework adapted to the Standard Model automatically gauge the B-L symmetry. The resulting Kaluza-Klein theory contains many nonSM fields, most of them only acting on flavour space. They can be removed like in the GR case. What remains is a B-L extension of the Standard Model with a complex scalar breaking the new symmetry.
\end{abstract}

Corfu Summer Institute 2019 "School and Workshops on Elementary Particle Physics and Gravity" (CORFU2019)

31 August - 25 September 2019

Corfù, Greece

${ }^{*}$ Speaker. 


\section{Introduction}

The Standard Model ${ }^{1}$ of particle physics is a highly sophisticated theoretical framework whose experimental success can hardly be exagerated. It contains subtle mechanisms (electroweak symmetry breaking, see-saw,...) and seemingly arbitrary inputs (gauge groups, representations, couplings,...) which have taken decades of work from the most brillant minds to uncover. The complexity of the end-product is such, that it has long been hoped to embed it in a simpler structure which would reveal itself at high energy. Grand unified theories are a possible approach, Superstring theory is another, more ambitious one, which includes gravity. The former has problematic predictions (proton decay), the latter has problems with making contact with the known low-energy regime. Both include supersymmetry, which has remained elusive despite intense search. In the 90's, a new perspective was opened by Alain Connes ${ }^{2}$. Thanks to a deep generalization of the notion of manifold, known as a Spectral Triple, in which the Dirac operator plays the role of (the inverse of) the metric, it has been possible to recast the Standard Model, at the classical level and in Euclidean signature, in a very simple form [2]. The advantages of this reformulation are its conceptual clarity and the natural apparition of most subtle features of the Standard Model which are normally added by hand. More precisely, the theory is formulated on a so-called almost-commutative manifold, which is so-to-speak a manifold with an additional discrete (and noncommutative) dimension. The presence of Higgs fields is then the immediate consequence of this additional dimension, in which they play the role of the inverse metric. Type I see-saw mechanism is also a generic feature of this kind of models. Moreover, one can lay down an action principle, the Connes-Lott action, which is of Yang-Mills type, and applies uniformly to all gauge and Higgs fields, which are thus completely unified within this framework. Later on, the Spectral Action principle has been formulated [3], which also includes the Einstein-Hilbert action for the manifold metric. This motivates the interpretation of the model as a kind of noncommutative Kaluza-Klein theory. Let us stress that the interest of the theory does not lie only on its elegance: it does have some practical consequences. In particular model-building is much more constrained in NCG than in usual gauge theory, thanks to the use of algebras instead of groups. One can add that the parameter space is also smaller than in the Standard Model, leading to predictions at high-energy, which can then be compared with experiment by running down the coupling constants to accessible scales. In fact, the first prediction of this kind including right-handed neutrinos turned out to give a mass to the Higgs boson which was 40 percent too high [4]. However, this prediction depended on the "big desert hypothesis", and it was later found that the inclusion of a single scalar field in this "desert" was enough to solve the problem [5]. A major trend in the recent years has been to find ways to accomodate for this new field in the formalism $[6,7,8]$.

In this paper we will quickly review the basics of the reformulation of the Standard Model as a noncommutative geometry, as well as the problems it meets, some of them already mentioned. We will be particularly interested in the version of the theory which includes gravity. This raises the question of general covariance and how it is implemented in the formalism. Equivalently, we are looking for a structure $\mathscr{B}$ which would represent the background, i.e. the bare differentiable manifold $M$, so that the addition of the Dirac operator to $\mathscr{B}$ would yield a Spectral Triple, like

\footnotetext{
${ }^{1}$ In this paper we define the Standard Model as including right-handed neutrinos and the see-saw mechanism.

${ }^{2}$ For a recent survey of the development of this line of thought, see [1].
} 
the addition of a metric to $M$ yields a Riemannian manifold. At first sight it seems that this is bound to fail, since the Dirac operator is not the only part of Spectral Triple which depends on the metric. Indeed, we will see by looking at the automorphism group that the naive attempt which consist of just removing the Dirac from a Spectral Triple does not work. However, the study of the automorphism group will give us an important clue which will lead to what seems to be an acceptable solution, at least in the cases of interest in physics. In brief we will define $\mathscr{B}$ to be a Spectral Triple deprived of its Dirac operator but enriched with a bimodule of noncommutative differential forms. We will call this new structure an algebraic background and show that in the case of manifolds as well as in the one of finite graphs, its automorphism group is exactly the expected one. However, and quite unexpectedly, a new kind of automorphism shows up in the case of the almost-commutative manifold of the Standard Model: $B-L$ gauge symmetry. This result is very encouraging, since it points to an extension of the Standard Model directly "from the inside of the formalism", and in total agreement with experimental input of a low Higgs mass. In other words, what we find is that, from this new point of view, the Standard Model is not consistent with NCG and has to be supplemented with at least a new $U(1)$-gauge symmetry and a scalar field. Such an extension has been widely discussed in the physics litterature. We will observe that it can be defined in conventional NCG, and seems in fact to be the only extension of the SM sitting in the Pati-Salam model in which the usual techniques of fluctuations of the Dirac operator still work.

\section{The NCG approach to particle physics in a nutshell}

The fundamental notion is that of a Spectral Triple. It is a tuple $(\mathscr{A}, \mathscr{H}, D)$, where $\mathscr{A}$ is a *-algebra, $\mathscr{H}$ a Hilbert space which bears a representation $\pi$ of $\mathscr{A}$, and $D$ is a selfadjoint operator on $\mathscr{H}$ called the Dirac operator. In even dimension there is also a chirality operator $\chi$, and in application to particle physics there is also an anti-linear operator $J$ called the real structure, which plays the role of charge conjugation and is related to the spin structure in the case of a manifold. We will always deal with real even spectral triples in this paper. The tuple $S=(\mathscr{A}, \mathscr{H}, \pi, D, \chi, J)$ has to satisfy some additional conditions. There are algebraic conditions on the operators $D, J$ and $\chi$ which are

- $D J=J D, D \chi=-\chi D$,

- $\chi^{2}=1,[\chi, \pi(a)]=0$ for all $a \in \mathscr{A}$,

- $J J^{\dagger}=1, J^{2}=\varepsilon$,

- $J \chi=\varepsilon^{\prime \prime} \chi J$.

where $\varepsilon, \varepsilon^{\prime \prime}$ are signs. The four possibilities on the pair $\left(\varepsilon, \varepsilon^{\prime \prime}\right)$ define an even integer modulo 8 called the KO-dimension according to table 1 .

Two other algebraic conditions are commonly assumed. To express them, the following notation will be useful: for every operator $T$ on $\mathscr{H}$ we write $T^{o}=J T^{\dagger} T^{-1}$. Then we can define

- the order 0 condition: $\left[\pi(a), \pi(b)^{o}\right]$ for all $a, b \in \mathscr{A}$, and

- the order 1 condition: $\left[[D, \pi(a)], \pi(b)^{o}\right]=0$ for all $a, b \in \mathscr{A}$. 


\begin{tabular}{|c||c|c|c|c|}
\hline$v$ & 0 & 2 & 4 & 6 \\
\hline$\varepsilon$ & 1 & -1 & -1 & 1 \\
\hline$\varepsilon^{\prime \prime}$ & 1 & -1 & 1 & -1 \\
\hline
\end{tabular}

Table 1: $\varepsilon, \varepsilon^{\prime \prime}$ in terms of KO-dimension $v$.

There are also some analytical conditions which we do not bother to write down as they will play little role in this paper ${ }^{3}$. A spectral triple satisfying all these conditions ${ }^{4}$ can be seen to be a noncommutative (dual) version of a Riemannian spin manifold [13]. In particular, if $\mathscr{A}$ is a commutative algebra, a topological space can be recovered by applying Gelfand duality to the completion of $\pi(\mathscr{A})$ in the operator norm. The points of this space are the pure states on the algebra, and Connes' formula

$$
d_{D}(\varphi, \psi)=\sup \{\mid \varphi(a)-\psi(a)\|a \in \mathscr{A},\|[D, a] \| \leq 1\}
$$

defines a generalized distance on it (generalized in the sense that it can take infinite values).

Spectral triples can be generalized to non-Euclidean signature, giving rise to Indefinite Spectral Triples (IST). This notion has already undergone several improvents $[14,15,16]$ and is still a work in progress. We will use the definition of [16] which takes into account the difficulty raised in [17]. A real even IST is a tuple $S=(\mathscr{A}, \mathscr{K}, \pi, D, \chi, J)$ containing the same objects as a spectral triple, submitted to the same conditions, with the following exceptions: the Hilbert space $\mathscr{H}$ is replaced with a pre-Krein space $\mathscr{K}$, i.e. a complex vector space equipped with a Hermitian form (.,.), which we often call a Krein product. The adjoint of an operator $T$ with respect to this form is written $T^{\times}$instead of $T^{\dagger}$. There are two additional signs $\kappa$ and $\kappa^{\prime \prime}$ defined by

$$
J^{\times}=\kappa J, \quad \chi^{\times}=\varepsilon^{\prime \prime} \kappa^{\prime \prime} \chi .
$$

These two signs depend on a new integer $\mu$ modulo 8 , called the metric dimension, according to table 2. The reason for the name is that it is really equal to $n \bmod 8$ when the IST is the canonical one associated to a manifold of dimension $n$, while the KO-dimension is equal to $p-q$ mod 8 , where $(p, q)$ is the signature. For more details on the KO-metric dimension pair, see [18]. Note that when $\varepsilon^{\prime \prime} \kappa^{\prime \prime}=1$, the two eigenspaces of $\chi$ are orthogonal with one another, while they are self-orthogonal when $\varepsilon^{\prime \prime} \kappa^{\prime \prime}=-1$. In the first case we say that the Krein product is even, and in second case that it is odd. This will play a key role when we come to tensor products.

\begin{tabular}{|c||c|c|c|c|}
\hline$\mu$ & 0 & 2 & 4 & 6 \\
\hline$\kappa$ & 1 & -1 & -1 & 1 \\
\hline$\kappa^{\prime \prime}$ & 1 & -1 & 1 & -1 \\
\hline
\end{tabular}

Table 2: Metric dimension in terms of $\mu$.

\footnotetext{
${ }^{3}$ They are related to the dimension and compactness/local compactness of the noncommutative manifold.

${ }^{4}$ Note that additional conditions have been explored which aim at characterizing more precisely the structure of the Standard Model triple. One can quote the second order condition [9, 10] or the Hodge condition [11, 12].
} 
A fundamental symmetry on a IST is a selfadjoint operator $\eta$ on $\mathscr{K}$, such that $\eta^{2}=1$ and $(., \eta$.) is positive-definite. A fundamental symmetry permits to define a norm and a Hilbert completion $\mathscr{K}_{\eta}$ of $\mathscr{K}$. In finite dimension the completion does not depend on $\eta$, so that one can define $\mathscr{K}$ by giving a Hilbert space and a fundamental symmetry, but this is no more true in general [17].

Let us now discuss the key notion of noncommutative 1-forms. Such a form is an operator $\omega \in \operatorname{End}(\mathscr{K})$ which can be written as

$$
\omega=\sum_{i} \pi\left(a_{i}\right)\left[D, \pi\left(b_{i}\right)\right]
$$

where the sum is finite and $a_{i}, b_{i} \in \mathscr{A}$. The vector space $\Omega_{D}^{1}$ of noncommutative 1 -forms is an $\mathscr{A}$-bimodule, and the derivation $d_{D}: \mathscr{A} \rightarrow \Omega_{D}^{1}, a \mapsto[D, \pi(a)]$ is a first-order differential calculus in the sense of [19]. One can try to extend $d_{D}$ to $\Omega_{D}^{1}$ by

$$
d_{D} \omega=\sum_{i}\left[D, \pi\left(a_{i}\right)\right]\left[D, \pi\left(b_{i}\right)\right]
$$

but a difficulty arises since $\omega$ can be decomposed as in (2.3) in several ways. This means that noncommutative 2-forms are defined modulo a certain ideal, called the junk, and that (2.4) makes sense modulo junk. We refer to [20] for more details. We can then define the curvature of a 1-form by

$$
\rho_{D}(\omega)=d_{D} \omega+\omega^{2} .
$$

The two most important classes of IST for application to particle physics are the canonical and almost-commutative ones. The canonical triple over a spin manifold is the paradigmatic example, the one that NCG seeks to generalize. Let us consider a semi-Riemannian manifold $M$ with metric $g$, which we suppose to be space and time-oriented and equipped with a spin structure $\sigma$. We recall that such a structure can be defined either in a topological way, through a covering of the principal frame bundle, or in the following algebraic way ${ }^{5}$, which is more convenient in noncommutative geometry (and in physics):

Definition 1. A spin structure on a space and time oriented manifold is a tuple $\sigma=(\mathscr{S}, \rho, \chi, H, J)$, where

1. $\mathscr{S}$ is a complex vector bundle over $M$,

2. $\rho: \mathbb{C l}(T M, g) \rightarrow \operatorname{End}(\mathscr{S})$ is a bundle isomorphism,

3. $\chi$, the chirality element, is the image under $\rho$ of the chirality element of the Clifford bundle corresponding to the orientation,

4. H is a positive spinor metric (see below),

5. $J$ is a bundle map $\mathscr{S} \rightarrow \mathscr{S}$, which is antilinear in the fibres, anticommutes with tangent vectors, and satisfies $J^{2}= \pm 1$, and $J^{\times} J= \pm 1$, where $J^{\times}$is the adjoint with respect to $H$.

\footnotetext{
${ }^{5}$ We refer to [9] for the equivalence of the two definitions.
} 
We need to explain what a positive spinor metric is: it is a smooth non-degenerate hermitian form $H$ on $\mathscr{S}$ such that for every tangent vector $v \in T_{x} M, \rho_{x}(v)$ is self-adjoint, and which satisfies a positivity condition. The latter is explained in full generality in [9]: let us just quote the two cases that will be of interest in this paper. In the Euclidean case, $H$ is asked to be positive-definite. In the anti-Lorentzian case ( $g$ of signature $(+,-, \ldots,-)), H_{x}(., \rho(v)$.) is asked to be positive-definite for every future-directed timelike tangent vector $v$. Note that in both cases this is a sign fixing: if $H$ is a spinor metric, either $H$ or $-H$ will be positive. For future use, let us observe that at each $x \in M$, $\operatorname{End}\left(\mathscr{S}_{x}\right)$ contains a copy of the group $\operatorname{Spin}(s, t)$, where $(s, t)$ is the signature of $g$, whose elements are defined by the following conditions:

(S1): $U \rho\left(T_{x} M\right) U^{-1} \subset \rho\left(T_{x} M\right)$,

(S2): $U$ is unitary for $H_{x}$,

(S3): $U$ commutes with $\chi_{x}$,

(S4): $U$ commutes with $J_{x}$.

The canonical IST over $(M, g, \sigma)$ is then defined by taking $\mathscr{A}$ to be the unitization $\tilde{\mathscr{C}}_{c}^{\infty}(M):=$ $\mathscr{C}_{c}^{\infty}(M) \oplus \mathbb{R}$ of the algebra of real smooth functions on $M$ with compact support, $\mathscr{K}$ to be the space $\Gamma_{c}^{\infty}(\mathscr{S})$ of smooth sections of $\mathscr{S}$ with compact support, equipped with the Krein product

$$
(\Phi, \Psi):=\int_{M} H_{x}\left(\Phi_{x}, \Psi_{x}\right) \operatorname{vol}_{g}
$$

and $\chi, J$ are the same objects as in the definition of $\sigma$ extended to sections of $\mathscr{S}$. Finally the Dirac operator is the canonical one associated with the metric and spin structure. When $g$ is Euclidean, Connes' distance formula (2.1) returns the geodesic distance on $M$. In the Lorentzian case there exists a generalization of this formula [21,22] if one assumes global hyperbolicity of $M$.

We now come to the second important class of examples, namely almost-commutative (indefinite) spectral triples. This is simply the tensor product of the canonical triple over a manifold with a finite-dimensional triple. The general rules for the tensor product of two IST are the following ones $[15,18]$. Suppose $S_{1}=\left(\mathscr{A}_{1}, \mathscr{K}_{1}, \ldots, J_{1}\right)$ and $S_{2}=\left(\mathscr{A}_{2}, \mathscr{K}_{2}, \ldots, J_{2}\right)$. We need to define $S=S_{1} \hat{\otimes} S_{2}:=(\mathscr{A}, \mathscr{K}, \ldots, J)$. The reason for using a graded tensor product is that the two spaces $\mathscr{K}_{i}, i=1,2$ are naturally graded by $\chi_{i}, i=1,2$, respectively. Hence we define $\mathscr{K}$ to be the vector space $\mathscr{K}=\mathscr{K}_{1} \otimes \mathscr{K}_{2}$ graded by $\chi=\chi_{1} \otimes \chi_{2}$. A vector $\psi$ in one of these spaces is given a grading $|\psi| \in \mathbb{Z}_{2}$, which is equal to 0 if it is in the +1 -eigenspace of the chirality. Similarly operators commuting with the chirality are said to be even and given the grading 0 , while operators anticommuting with it are said to be odd and given the grading 1. Even or odd operators are said to be homogenous. For homogenous operators $T_{i} \in \operatorname{End}\left(\mathscr{K}_{i}\right), i=1,2$, we can define the graded tensor product $T_{1} \hat{\otimes} T_{2}$ by

$$
\left(T_{1} \hat{\otimes} T_{2}\right)\left(\psi_{1} \otimes \psi_{2}\right):=(-1)^{\left|\psi_{1}\right|\left|T_{2}\right|} T_{1} \psi_{1} \otimes T_{2} \psi_{2}
$$

The graded tensor product of homogenous operators is related to the usual tensor product by the formula $T_{1} \hat{\otimes} T_{2}=T_{1} \chi_{1}^{\left|T_{2}\right|} \otimes T_{2}$. The algebra $\mathscr{A}$ is simply $\mathscr{A}=\mathscr{A}_{1} \otimes \mathscr{A}_{2}$ (the algebraic tensor product 
will suffice for our purpose), and the representation $\pi$ is $\pi_{1} \otimes \pi_{2}$ (which is also a graded tensor product since both factors are even). The real structure $J$ is $J_{1} \chi_{1}^{\left|J_{2}\right|} \hat{\otimes} J_{2} \chi_{2}^{\left|J_{2}\right|}$. The Dirac operator is

$$
D=D_{1} \hat{\otimes} 1+1 \hat{\otimes} D_{2} .
$$

Finally we need to define the Krein product on $\mathscr{K}$. It is given by

$$
\left(\phi_{1} \hat{\otimes} \phi_{2}, \psi_{1} \hat{\otimes} \psi_{2}\right)=\left(\phi_{1}, \psi_{1}\right)_{1}\left(\phi_{2}, \beta \psi_{2}\right)_{2},
$$

where $\beta=1$ if $(., .)_{1}$ is even, $\beta=\chi_{2}$ if $(., .)_{1}$ is odd and $(., .)_{2}$ is even, and $\beta=i \chi_{2}$ if $(., .)_{1,2}$ are both odd. The justifications for the less obvious parts of these rules can be found in [18]. With them, the $\mathrm{KO}$ and metric dimensions are additive.

The Euclidean Standard Model triple is the almost-commutative triple $S=S_{M} \hat{\otimes} S_{F}$ where $S_{M}$ is the canonical triple over the Euclidean four-dimensional spacetime, and $S_{F}=\left(\mathscr{A}_{F}, \mathscr{H}_{F}, \pi_{F}, D_{F}, J_{F}, \chi_{F}\right)$ is a finite-dimensional triple which we now define. The algebra $\mathscr{A}_{F}$ is taken to be

$$
\mathscr{A}_{F}=\mathbb{C} \oplus \mathbb{H} \oplus M_{3}(\mathbb{C})
$$

This choice is imposed by the gauge group of the Standard Model. The Hilbert space $\mathscr{H}_{F}$ is also an experimental input. It is of the form

$$
\mathscr{H}_{F}=\mathscr{H}_{R} \oplus \mathscr{H}_{L} \oplus \mathscr{H}_{\bar{R}} \oplus \mathscr{H}_{\bar{L}},
$$

where each $\mathscr{H}_{\sigma}, \sigma=R, \ldots, \bar{L}$ is $\mathscr{H}_{0}:=\mathbb{C}^{2} \otimes \mathbb{C}^{4} \otimes \mathbb{C}^{3}$ equipped with the canonical scalar product. The canonical basis vectors of $\mathscr{H}_{0}$ are identified with elementary fermions in the following way: the $\mathbb{C}^{2}$ factor represents the weak isospin space, with canonical basis $(u, d)$ (up,down), the $\mathbb{C}^{4}$ is the color space, seeing leptons as a fourth colour as in the Pati-Salam model, with basis $(\ell, r, g, b)$, and the $\mathbb{C}^{3}$ is the generation space with basis $(1,2,3)$. For instance the vector basis $u \otimes \ell \otimes 3$ is interpreted as a tau neutrino. Moreover, vectors in $\mathscr{H}_{R}$ are interpreted as right-handed particles, vectors in $\mathscr{H}_{\bar{L}}$ as left-handed anti-particles and so on. Very often, we will need to decompose $\mathbb{C}^{4}$ into $\mathbb{C} \oplus \mathbb{C}^{3}$. Since the quark colour and generation spaces have the same dimension, we will write them $\mathbb{C}_{q}^{3}$ and $\mathbb{C}_{g}^{3}$ to avoid any confusion.

The representation $\pi$ is defined as follows. For an element $(\lambda, q, m) \in \mathbb{C} \oplus \mathbb{H} \oplus M_{3}(\mathbb{C})$, one defines

$$
\pi(\lambda, q, m)=\operatorname{diag}\left(q_{\lambda} \otimes 1_{4} \otimes 1_{3}, q \otimes 1_{4} \otimes 1_{3}, 1_{2} \otimes(\lambda \oplus m) \otimes 1_{3}, 1_{2} \otimes(\lambda \oplus m) \otimes 1_{3}\right)
$$

where $q_{\lambda}=\left(\begin{array}{ll}\lambda & 0 \\ 0 & \bar{\lambda}\end{array}\right)$ is the embedding of $\mathbb{C}$ into $\mathbb{H}$ seen as the algebra of matrices of the form $\left(\begin{array}{cc}\alpha & \beta \\ -\bar{\beta} & \bar{\alpha}\end{array}\right)$. Moreover, $\lambda \oplus m$ is the block diagonal matrix $\left(\begin{array}{cc}\lambda & 0 \\ 0 & m\end{array}\right)$ acting on the colour $\mathbb{C}^{4}$. The chirality operator is

$$
\chi_{F}=\operatorname{diag}(1,-1,-1,1)
$$

where 1 is the identity operator on $\mathscr{H}_{0}$, and the real structure is

$$
J_{F}=\left(\begin{array}{cccc}
0 & 0 & 1 & 0 \\
0 & 0 & 0 & 1 \\
1 & 0 & 0 & 0 \\
0 & 1 & 0 & 0
\end{array}\right) \text { oc.c. }
$$


with the same notation and c.c. means complex conjugation. It is easy to see that $S_{F}$ and $S$ both satisfy the order 0 condition. The commutation with $J_{F}$, anticommutation with $\chi_{F}$, self-adjointness, and the first-order condition force the finite Dirac $D_{F}$ to have the form

$$
D_{F}=\left(\begin{array}{cccc}
0 & Y^{\dagger} & M^{\dagger} & 0 \\
Y & 0 & 0 & 0 \\
M & 0 & 0 & Y^{T} \\
0 & 0 & \bar{Y} & 0
\end{array}\right)
$$

where

$$
Y=\left(\begin{array}{cc}
Y_{\ell} & 0 \\
0 & 1_{3} \otimes Y_{q}
\end{array}\right), Y_{\ell}, Y_{q} \in M_{2}\left(M_{3}(\mathbb{C})\right)
$$

and

$$
M=\left(\begin{array}{llll}
a & b^{T} & x^{T} & 0 \\
b & 0 & y^{T} & 0 \\
x & y & 0 & 0 \\
0 & 0 & 0 & 0
\end{array}\right), a, b \in M_{3}(\mathbb{C}), a^{T}=a, c, d \in M_{2,3}\left(M_{3}(\mathbb{C})\right)
$$

Understanding (2.16) and (2.17) needs a little explanation. The matrix in (2.16) is written in the block form induced by writing $\mathscr{H}_{0}$ as $\left(\mathbb{C} \oplus \mathbb{C}_{q}^{3}\right) \otimes \mathbb{C}^{2} \otimes \mathbb{C}_{g}^{3}$. Hence $Y_{\ell}$ and $Y_{q}$ act on the space $\mathbb{C}^{2} \otimes \mathbb{C}_{g}^{3}$ while $1_{3}$ acts on $\mathbb{C}_{q}^{3}$. Matrix $M$ has been written by decomposing $\mathscr{H}_{0}$ into neutrinos $\oplus$ electrons $\oplus$ up quarks $\oplus$ down quarks. More precisely $\mathbb{C}^{2} \otimes\left(\mathbb{C} \oplus \mathbb{C}_{q}^{3}\right)$ is first written as $\mathbb{C}^{2} \oplus \mathbb{C}^{2} \otimes \mathbb{C}_{q}^{3}$ and then each factor $\mathbb{C}^{2}$ is decomposed using the $(u, d)$ basis. Each matrix element of $M$ acts on the generation space.

This very special form of $D_{F}$ is already an interesting result: there are many blocks of zeros in $D_{F}$, and if some of them did not vanish they would be responsible for couplings which do not exist in the Standard Model. However, other unwanted couplings could still be introduced by arbitrary matrices $Y_{\ell}, Y_{q}$ and $a, b, x, y$. Thus, we must further assume that these matrices have the following special form:

$$
Y_{\ell}=\left(\begin{array}{cc}
Y_{v} & 0 \\
0 & Y_{e}
\end{array}\right), Y_{q}=\left(\begin{array}{cc}
Y_{u} & 0 \\
0 & Y_{d}
\end{array}\right),
$$

where we have decomposed the $\mathbb{C}^{2}$ factor using the $(u, d)$ basis, while $b=x=y=0$, which means that

$$
M=\left(\begin{array}{ll}
1 & 0 \\
0 & 0
\end{array}\right) \otimes\left(\begin{array}{cc}
m & 0 \\
0 & 0
\end{array}\right)
$$

Some work have been done to derive (2.18) and (2.19) from general principles, like the massless photon condition [23], or the second-order condition [9, 10]. The physical interpretation of the entries of $D_{F}$ is now that $Y_{v}, Y_{e}, Y_{v}, Y_{e}$ are the Dirac mass matrices ${ }^{6}$ while $m$, which is symmetric since $M$ is, is the Majorana mass matrix for right-handed neutrinos, responsible for the usual (type I) see-saw mechanism.

In the anti-Lorentzian case, a few changes are in order. The manifold is now of signature $(+,-,-,-)$, with the consequence that the spinor metric is odd. Formula (2.9) then imposes a modification of the finite Hermitian form also. The finite triple $S_{F}$ thus has the same algebra,

\footnotetext{
${ }^{6}$ More precisely, there is a multiplicative constant.
} 
representation, chirality, and vector space $\mathscr{H}_{F}$, but the latter is now equipped with the Krein product $(., .)_{F}:=\left\langle., \chi_{F}.\right\rangle$, where $\langle.,$.$\rangle is the canonical scalar product { }^{7}$. The real structure is also changed to

$$
J_{F}=\left(\begin{array}{cccc}
0 & 0 & -1 & 0 \\
0 & 0 & 0 & -1 \\
1 & 0 & 0 & 0 \\
0 & 1 & 0 & 0
\end{array}\right) \text { oc.c. }
$$

and, in order to be compatible with the new choices of Hermitian form and real structure, the finite Dirac becomes

$$
D_{F}=\left(\begin{array}{cccc}
0 & -Y^{\dagger} & -M^{\dagger} & 0 \\
Y & 0 & 0 & 0 \\
M & 0 & 0 & -Y^{T} \\
0 & 0 & \bar{Y} & 0
\end{array}\right)
$$

where $Y$ and $W$ retain their particular forms.

The bosonic fields are introduced through so-called "fluctuations of the Dirac operator". Given a selfadjoint 1-form $\omega$, one defines the fluctuated Dirac operator

$$
D_{\omega}:=D+\omega+\omega^{o}
$$

It is interesting to note that one could replace $D$ with $D_{\omega}$ in the definition of the spectral triple: all the axioms would still be satisfied and the bimodule of 1-forms would be the same, thanks to the order 1 condition. The introduction of these fluctuations can be justified by the notion of Morita self-equivalence of the algebra, which in more traditional terms means that the replacement of $D$ by $D_{\omega}$ is equivalent to the replacement of the usual derivative with the covariant one. Thus, the bosonic configuration space of the noncommutative Standard Model is

$$
\mathscr{D}_{\text {fluct }}:=\left\{D_{\omega} \mid \omega \in \Omega_{D}^{1}, \omega^{\dagger}=\omega\right\} .
$$

This space is invariant under gauge transformations, which are defined by

$$
\Upsilon(u):=\pi(u) J \pi(u) J^{-1},
$$

$u$ being a unitary element of $\mathscr{A}$. Under such a transformation we have $D_{\omega} \mapsto U D_{\omega} U^{-1}=D_{\omega^{u}}$, where

$$
\omega^{u}=\pi(u)\left[D, \pi(u)^{-1}\right]+\pi(u) \omega \pi(u)^{-1}
$$

Moreover the curvature (2.5) is gauge-covariant, i.e.

$$
\rho_{D}\left(\omega^{u}\right)=\pi(u) \rho_{D}(\omega) \pi(u)^{-1} .
$$

Gauge-transformations in the sense of NCG are easily seen to be in bijective correspondance with usual gauge transformations with local gauge group $U(1) \times S U(2) \times U(3)$. We see that this group is not exactly the one of the Standard Model, having $U(3)$ instead of $S U(3)$. The effect is the appearance through (2.24) of an extra $U(1)$ gauge field, which we call $X$. This field can be removed

\footnotetext{
${ }^{7}$ The finite Krein product is different whether we consider fermionic variables to be commuting or anti-commuting. Our choice corresponds to the anti-commuting case. For details on this issue, see [15].
} 
by restricting by hand the gauge transformation to those which satisfy $\operatorname{det}(\pi(u))=1$ : this is the unimodularity condition, which turns out to be equivalent to the cancellation of quantum anomalies.

The fermionic fields are simply elements of the Hilbert space $\mathscr{H}=\mathscr{H}_{M} \otimes \mathscr{H}_{F}$ of the total triple $S$. In other words they are sections of the bundle $\mathscr{S} \otimes \mathscr{H}_{F}$. Since the information about chirality and charge is contained both in the spinor space and $\mathscr{H}_{F}$, there is a quadruplication of the fermionic degrees of freedom [24]. In anti-Lorentzian signature this problem can be fixed by restricting the fermionic fields to the subspace $\mathscr{H}_{\text {MW }}$ defined by the "Majorana-Weyl conditions"

$$
\begin{aligned}
& J \Psi=\Psi \\
& \chi \Psi=\Psi .
\end{aligned}
$$

These conditions were introduced in [25] and later found to be unique up to phases under some symmetry assumptions [26]. The non-triviality of the space $\mathscr{H}_{\mathrm{MW}}$ imposes that $J^{2}=1$ and $J \chi=\chi J$, which fixes the KO-dimension of the total triple to be 0 . If $M$ has anti-Lorentzian signature, the canonical triple has KO-dimension $1-3=-2 \bmod 8$, and this requires the finite triple to have KO-dimension $2 \bmod 8$, and in particular that $J_{F}^{2}=-1$. This explains the choice (2.20). Now it can be shown that $M$ is a symmetric matrix when $J_{F}$ squares to -1 , and an anti-symmetric one when it squares to 1 . It thus turns out that the resolution of the fermion doubling problem by (2.27) is tied to type I see-saw mechanism, as observed in [25]. A similar phenomenon exists in Euclidean signature [27].

The fermionic action is

$$
S_{f}\left(D_{\omega}, \Psi\right)=\left\langle J \Psi, D_{\omega} \Psi\right\rangle
$$

in the Euclidean case and

$$
S_{f}\left(D_{\omega}, \Psi\right)=\left(\Psi, D_{\omega} \Psi\right),
$$

in the anti-Lorentzian one. It reproduces all the fermionic terms of the SM, including the Majorana mass terms for right-handed neutrinos.

The first bosonic action to be defined was that of Connes and Lott [2]. It is equal to

$$
S_{b}^{\mathrm{CL}}(\omega)=\int_{M} \operatorname{Tr}\left(z P\left(\rho_{D}(\omega)\right)^{2}\right) \operatorname{vol}_{g},
$$

where $P$ is the operator which orthogonally projects away from the space of junk 2-forms (the scalar product on operators is the Hilbert-Schmidt one) and $z$ is a normalization factor which here is simply a real number (but see below). This is none other than a Yang-Mills action, but it contains in a single formula all the bosonic interaction and kinetic terms of the Standard Model, including those of the Higgs. This action is invariant under gauge-transformations thanks to (2.26) and a similar property for $P$. In anti-Lorentzian signature (2.30) continues to make sense on the condition that the orthogonal projector $P$ is well-defined, which it is, since the restriction of the Krein-Schmidt product to the junk space is non-degenerate $[15,28]$.

Another possibility, which is to date available only for Euclidean signature, is the Spectral Action of Connes-Chamseddine [29]:

$$
S_{b}^{\mathrm{CC}}\left(D_{\omega}\right)=\operatorname{Tr}\left(\chi\left(\frac{D_{\omega}^{2}}{m_{0}^{2}}\right)\right)
$$


Here $m_{0}$ is a mass parameter and $\chi$ is a smooth approximation of the characteristic function of the interval $[0,1]$. At first sight it would seem to introduce an infinite arbitrariness in the definition of the action, but in fact it can be shown that the spectral action only depends on $\chi$ only through

$$
c_{0}:=\int_{0}^{\infty} \chi(u) d u, c_{2}:=\int_{0}^{\infty} \chi(u) d u, \text { and } \chi(0) .
$$

Since the spectral action only depends on the spectrum of $D_{\omega}$, it is clear that it is not only gaugeinvariant, but invariant under any unitary operator ! The spectral action can be expanded into powers of $\frac{1}{m_{0}}$ as

$$
S_{b}^{\mathrm{CC}}\left(D_{\omega}\right)=m_{0}^{4} c_{0} a_{0}\left(D_{\omega}^{2}\right)+m_{0}^{2} c_{2} a_{2}\left(D_{\omega}^{2}\right)+\chi(0) a_{4}\left(D_{\omega}^{2}\right)+\mathscr{O}\left(m_{0}^{-2}\right) .
$$

The full derivation of the coefficients $a_{0}, a_{2}, a_{4}$ can be found in [30], explained in a pedagogical way. For our purpose, it will be sufficient to report that $S_{b}^{\mathrm{CC}}$ can be re-expressed as

$$
S_{b}^{\mathrm{CC}}\left(D_{\omega}\right)=S_{\mathrm{EH}}(g)+S_{\mathrm{HO}}(g)+S_{\mathrm{SM}}(\omega)+S_{\mathrm{HM}}(g, H)+\mathscr{O}\left(m_{0}^{-2}\right),
$$

where $S_{\mathrm{EH}}(g)$ is the Einstein-Hilbert action of $g, S_{\mathrm{HO}}(g)$ contains higher-order curvature terms, $S_{\mathrm{SM}}(\omega)$ is the bosonic action of the Standard Model, and $S_{\mathrm{HM}}(g, H)$ is a coupling between the Higgs and the scalar curvature. Note that the scalar curvature term has a coefficient which is of order $m_{0}^{2}$ while the cosmological constant term is of order $m_{0}^{4}$. In order to obtain the correct numerical value for Newton's constant, one has to set $m_{0}$ to the Planck scale, which produces an enormous cosmological constant.

Whether one uses the Connes-Lott or the spectral action, there are less free parameters than in the usual approach to the Standard Model. In particular, one gets the relation

$$
g_{3}^{2}=g_{2}^{2}=\frac{5}{3} g_{1}^{2}
$$

between the gauge couplings which is the same as in Grand Unified Theories. There is also a mass relation of the form

$$
\text { sum of fermion masses squared }=k \times \mathrm{W} \text { boson mass squared }
$$

where the constant $k$ depends on the action chosen. This indicates that the Connes-Lott as well as the spectral action is valid at high energy only. In order to make predictions at accesible energies, one runs down the coupling from some chosen unification scale, assuming the big desert. One obtain a Higgs mass of about $170 \mathrm{GeV}$ [4] with the spectral action. With the Euclidean ConnesLott action and without right-handed neutrino, the estimate $223<m_{\text {Higgs }}<304 \mathrm{GeV}$ can be found in [31]. Another way to get predictions with the latter model (which could also work with the spectral action but has never been attempted as far as we know), is to replace the normalization factor $z$ in (2.30) with an operator [32], which thus plays the role of a noncommutative gauge coupling. Obviously $z$ has to satisfy some constraints in order for (2.30) to remain gauge-invariant. If $z$ is asked to commute with the Dirac operator, the estimate $m_{\mathrm{Higgs}}=280 \pm 33 \mathrm{GeV}$ can be found $[33,34]$. However there is no compelling reason to ask for this, and without this hypothesis the lower bound is removed [35]. However it seems to us that the conditions on $z$ would need to be reanalyzed in the context of algebraic backgrounds. Let us stress once again that all the predictions 
found in the literature using the Connes-Lott action date from a period when the right-handed neutrinos had not been added to the model. It can be shown that including right-handed neutrinos makes the renormalization group prediction go down to a value in the $175-200 \mathrm{GeV}$ interval.

As we see, all the predictions of the noncommutative Standard Model, using various techniques, came up with a too large mass for the Higgs particle. For the renormalization group predictions it could simply mean that the big desert hypothesis is not correct. In fact it turns out that a single scalar field is enough to "cure" the prediction $[5,30,36]$.

In summary, we have seen in this section that the bosonic configuration space $\mathscr{D}_{\text {fluct }}$ and the fermionic configuration space $\mathscr{H}_{\mathrm{MW}}$ equipped with the fermionic action and the Connes-Lott bosonic action define a noncommutative gauge theory which reproduces the Standard Model (up to an anomalous $U(1)$-gauge field which can be removed by the unimodularity condition) with righthanded neutrinos and type I see-saw mechanism, both in Euclidean and anti-Lorentzian signature. In Euclidean signature one can use also the spectral action. In both cases, one obtains a theory which has less free parameters than the Standard Model and yields to predictions including a mass for the Higgs boson which is too high.

\section{Noncommutative Geometry and general covariance}

Thanks to equation (2.34), the spectral action is often interpreted as gravitational in nature, and the spectral SM as a form of Kaluza-Kkein theory defined on a noncommutative spacetime. In order for this interpretation to be correct, the usual gravitational degrees of freedom should be present in the configuration space $\mathscr{D}_{\text {fluct }}$, but they are not. In fact, the metric $g$ is just a constant in (2.34). Of course it is very tempting to enlarge the configuration space in order to let $g$ vary, since in that case the spectral action would yield the same dynamics as GR, at least to leading order. To do so consistently, one would also have to implement the symmetry group of GR in the spectral triple formalism. This raises the question of the definition of symmetries in this context.

The most direct attempt consists simply of removing the Dirac operator from the definition of a spectral triple in order to make it dynamical. For the sake of the argument, let us define a pre-spectral triple to be exactly the same thing as a spectral triple except it has no fixed Dirac operator. Instead, given a pre-spectral triple $\mathscr{T}$, we define the bosonic configuration space of $\mathscr{T}$ to be the space of all operators $D$ such that $(\mathscr{T}, D)$ is a spectral triple. The idea is to think of $\mathscr{T}$ as the background while $D$ is the dynamical part ${ }^{8}$. The automorphism group of $\mathscr{T}$ then consists of all the operators which leave invariant the background structure, namely the operators on $\mathscr{K}$ which:

- are unitary,

- commute with $\chi$ and $J$,

- leave $\pi(\mathscr{A})$ invariant (i.e. $U \pi(\mathscr{A}) U^{-1}=\pi(\mathscr{A})$.

In the best of worlds, the objects $\mathscr{T}, D$ and $\operatorname{Aut}(\mathscr{T})$ would correspond in the commutative case to the manifold, metric, and diffeomorphism group. However, the true situation is much more entangled. To begin with, one needs the Dirac operator in the reconstruction theorem in order to

\footnotetext{
${ }^{8}$ Similar interpretations of NCG have surfaced several times over the years [37, 38, 39].
} 
recover the differential structure on the manifold. Suppose we ignore this problem for the moment, saying that we believe in the idea strongly enough to go on without a reconstruction theorem in our pocket. After all we do not really need it to apply the idea in physics. We then meet the related problem of constructing a "canonical pre-spectral triple" out a bare differential manifold with no background metric. Indeed, in the construction of the canonical triple, the metric appears not only in the definition (2.6) of the Krein product of spinor fields, but also in the very definition of spin structure. However, a working solution can be found, and will enable us to test our ideas. Let us consider a parallelizable manifold $M$. We consider it to be 4-dimensional for simplicity's sake, though this does not play an essential role (see [16] for the general case). We let $S=\mathbb{C}^{4}$ be the standard spinor space, and choose gamma matrices $\gamma_{a}, a=0, \ldots, 3$. It is important that $\gamma_{a}$ be either selfadjoint or anti-selfadjoint, as is the case in Dirac or Weyl representation. The spinor metric on $S$ is defined to be $H_{S}\left(\psi, \psi^{\prime}\right)=\psi^{\dagger} \gamma_{0} \psi^{\prime}$. We let $\mathscr{K}_{M}$ be the space of compactly supported spinor fields, which are just $S$-valued functions since the spinor bundle is here trivial, and $\mathscr{A}_{M}=\tilde{\mathscr{C}}_{c}^{\infty}(M)$ just as before, with the usual representation by multiplication which we denote by $\pi_{M}$. We need a volume form and for this we choose an origin metric $g$ on $M$ and define the Krein product by (2.6), with $H_{x}=H_{S}$. This is the only place where $g$ will play a role. It would seem that we are putting a volume form in the background and entering the field of unimodular gravity. We will see below that it is in fact not the case, the reason being that it is possible for an operator to preserve the Krein product on $\mathscr{K}_{M}$ without preserving $H_{S}$ and $\operatorname{vol}_{g}$ separately. Finally we let $\chi_{M}$ and $J_{M}$ be the operators on $\mathscr{K}_{M}$ defined respectively by

$$
\left(\chi_{M} \Psi\right)_{x}=\gamma_{5} \Psi_{x},\left(J_{M} \Psi\right)_{x}=\gamma_{2} \bar{\Psi}_{x},
$$

where bar means complex conjugation in the canonical basis of $S$. We can easily check that we have defined an indefinite pre-spectral triple $\mathscr{T}_{M}=\left(\mathscr{A}_{M}, \mathscr{K}_{M}, \pi_{M}, \chi_{M}, J_{M}\right)$, and we now wonder about the Dirac operators. First we consider a globally defined frame field (or tetrad) $e=\left(e_{a}\right)_{a=0, \ldots, 3}$ (this is where the parallelizability hypothesis is used). Observe that $e$ defines at the same time a metric $g_{e}$, which is the unique anti-Lorentzian metric such that $e$ is a pseudo-orthonormal basis, with $e_{0}$ timelike. The non-vanishing timelike vector field $e_{0}$ defines a time-orientation, and the tetrad $e$ is positive for a unique spacetime orientation. Thus $M$ equipped with $e$ becomes a space and time oriented anti-Lorentzian manifold, and the choices we have made above determine a compatible spin structure $\sigma_{e}$ in the sense of definition 1, with $\mathscr{S}=M \times S, H=H_{S}, \chi$ and $J$ defined by (3.1), and $\rho$ uniquely defined by

$$
\rho\left(e_{a}\right)=\gamma_{a}
$$

Now the metric $g_{e}$ and compatible spin structure $\sigma_{e}$ also provide the canonical Dirac operator $D(e)$, defined in formula by

$$
D(e)=i \sum_{a} g\left(e_{a}, e_{a}\right) \gamma_{e} \nabla_{e_{a}}^{\sigma_{e}}
$$

where $\nabla_{e_{a}}^{\Sigma_{e}}$ is the unique spin connection associated with the spin structure $\sigma_{e}$. Hence, for every $e$, the pair $\left(\mathscr{T}_{M}, D(e)\right)$ is an indefinite spectral triple. The configuration space of $\mathscr{T}_{M}$ thus contains a subset which is in bijective correspondence with the set of all tetrads on $M$, that is the configuration space of tetradic gravity. However we will see in section 4 below that it contains other fields. We will nevertheless consider the above construction to be on the right track and now inquire about the automorphism group of $\mathscr{T}_{M}$. 
To begin with, let $\theta$ be diffeomorphism of $M$ and define $U_{\theta}: \mathscr{K}_{M} \rightarrow \mathscr{K}_{M}$ by

$$
\left(U_{\theta} \Psi\right)_{x}=\sqrt{\frac{\operatorname{vol}_{\theta_{*} g}}{\operatorname{vol}_{g}}} \Psi_{\theta^{-1}(x)} .
$$

In other words, $U_{\theta} \Psi$ is just the pullback of $\Psi$ by $\theta$ modified by a factor which exactly compensates the Jacobian appearing in the integral (2.6) with the change of variable $y=\theta(x)$. This ensures that $U_{\theta}$ is unitary (and explain why the origin metric $g$ is not a background object). Let us now consider a smooth map $\Sigma: M \rightarrow \operatorname{Spin}(1,3)$, where $\operatorname{Spin}(1,3)$ is seen as the subset of $\operatorname{End}(S)$ defined by the conditions $(S 1), \ldots,(S 4)$ of section 2 . The we define $U_{\Sigma}: \mathscr{K}_{M} \rightarrow \mathscr{K}_{M}$ by

$$
\left(U_{\Sigma} \Psi\right)_{x}=\Sigma_{x} \Psi_{x}
$$

Now let us recall that in tetradic GR there are exacty two kinds of symmetry: diffeomorphisms and "local Lorentz transformations" (LLT), which really are Lorentz transformations of the tangent space lifted to the spin group and acting on spinors, and are none other than the $U_{\Sigma}$ we have just defined. Since $U_{\Sigma}$ and $U_{\theta}$ are easily seen to be automorphisms of the pre-spectral triple $\mathscr{T}_{M}$, the symmetry group $\operatorname{Aut}\left(\mathscr{T}_{M}\right)$ contains the symmetry group $\operatorname{Diff}(M) \ltimes \Gamma(\operatorname{Spin}(1,3))$ of tetradic GR. To see if it is equal to it, we consider an element $U$ of $\operatorname{Aut}\left(\mathscr{T}_{M}\right)$. Acting with an automorphism $U_{\theta}$, we can suppose without loss of generality that $U$ commutes with the algebra elements, and is thus an operator of the form $(U \Psi)_{x}=U_{x} \Psi_{x}$, acting locally on spinors. Moreover, by definition of the autormorphisms of a pre-spectral triple, $U_{x}$ must be unitary for $H_{S}$ and commute with $\chi_{x}$ and $J_{x}$. It thus satisfies the conditions $(S 2),(S 3)$ and $(S 4)$. It turns out that in dimension $\leq 4$, condition $(S 1)$ is redundant and can be omitted. Thus we see that the automorphism group of $\mathscr{T}_{M}$ is, by a happy coincidence, exactly the symmetry group of tetradic GR ! In fact it is not difficult to display a counterexample if we redo the construction of $\mathscr{T}_{M}$ in dimension 6 and signature $(1,5)$. The operator defined by $(U \Psi)_{x}=U_{x} \Psi_{x}$ where $U_{x}$ is constant and equal to $(\sinh t) \gamma_{0} \gamma_{1}+(\cosh t) \gamma_{2} \gamma_{3} \gamma_{4} \gamma_{5}$, for any real and non-zero $t$, is easily seen to be a pre-spectral triple automorphism. However, since $U_{x}$ does not satisfy $(S 1)$, it is not in the group generated by diffeomorphisms and LLT.

This example can make us suspect that our notion of automorphism is too general: we lack a condition, which means that we lack a background structure in pre-spectral triples. In order to identify which one, let us consider condition $(S 1)$ in the present context. It tells us that the subspace $\Gamma$ of $S$ spanned by the gamma matrices must be preserved by the local operators acting on spinors. Now observe that, whatever the tetrad e, the bimodule of noncommutative 1-forms $\Omega_{D(e)}^{1}$ is always equal to the bimodule of $\Gamma$-valued fields, and that this bimodule is indeed preserved by diffeomorphisms and LLT in every dimension. We are thus led to the conclusion that in going from spectral triples to pre-spectral triples by removing $D$, we removed too much. We should instead replace $D$ by the bimodule $\Omega_{D}^{1}$. If we do that in the case of a manifold, we obtain a structure which has exactly the symmetry group of tetradic GR in every dimension. This leads us to the definition of algebraic backgrounds in the next section.

\section{Algebraic backgrounds}

Motivated by the examples of the previous section, we define the following structure. 
Definition 2. An algebraic background is a tuple $\mathscr{B}=\left(\mathscr{A}, \mathscr{K}, \pi, \chi, J, \Omega^{1}\right)$ where:

1. $\mathscr{K}$ is a pre-Krein space,

2. The antilinear operator $J$ and the linear operator $\chi$ satisfy:

$$
\chi^{2}=1, \quad J^{2}=\varepsilon, \quad J \chi=\varepsilon^{\prime \prime} \chi J, \quad J^{\times}=\kappa J, \quad \chi^{\times}=\varepsilon^{\prime \prime} \kappa^{\prime \prime} \chi
$$

3. $\mathscr{A}$ is a $*$-algebra and $\pi$ is a $*$-representation of it on $\mathscr{K}$,

4. the chirality operator $\chi$ commutes with $\pi(a)$ for all $a \in \mathscr{A}$,

5. the "bimodule of 1-forms" $\Omega^{1}$ is an $\mathscr{A}$-bimodule of operators on $\mathscr{K}$ such that for any $\omega \in$ $\Omega^{1}, \omega \chi=-\chi \omega$.

In short, an algebraic background is a pair $\left(\mathscr{T}, \Omega^{1}\right)$ where $\mathscr{T}$ is a pre-spectral triple and $\Omega^{1}$ is an odd bimodule. There are some boundedness conditions which we have not given. On the contrary, we have added the condition that $\mathscr{A}$ is a $*$-algebra and $\pi$ a $*$-representation, which might be too stong in some contexts. We refer to [16] for details on these matters.

Given an algebraic background $\mathscr{B}=\left(\mathscr{T}, \Omega^{1}\right)$, we define a compatible Dirac operator on $\mathscr{B}$ to be an operator $D$ such that:

1. $(\mathscr{T}, D)$ is a spectral triple,

2. $\Omega_{D}^{1} \subset \Omega^{1}$.

If the inclusion in the second condition is an equality, we say that $D$ is regular. The vector space of all compatible Dirac operators on $\mathscr{B}$ is called the configuration space of $\mathscr{B}$. We will always want the configuration space to be non-empty, and most often ask that it contains at least a regular element. In that case, the bimodule $\Omega^{1}$ and the derivation $d_{D}: \mathscr{A} \rightarrow \Omega^{1}$ defined by $d_{D} a=[D, \pi(a)]$ is a first-order differential calculus (FODC) in the sense of [19].

We are now in a position to compare the automorphisms of the three structures which have emerged in our discussion, namely pre-spectral triples, algebraic backgrounds and spectral triples. Let $\mathscr{T}=(\mathscr{A}, \mathscr{K}, \pi, J, \chi)$ be a pre-spectral triple, $\mathscr{B}=\left(\mathscr{T}, \Omega^{1}\right)$ be an algebraic background, and $S=(\mathscr{T}, D)$ be a spectral triple. Then, as already explained in section 3 , an operator $U$ on $\mathscr{K}$ is an automorphism of $\mathscr{T}$ iff

1. $U$ is unitary,

2. $U$ commutes with $\chi$ and $J$,

3. $U \pi(\mathscr{A}) U^{-1}=\pi(\mathscr{A})$.

It is an automorphism of $\mathscr{B}$ if in addition it satisfies

4. $U \Omega^{1} U^{-1} \subset \Omega^{1}$ 
Finally, $U$ is an automorphism of $S$ if it is an automorphism of $\mathscr{T}$ and commutes with $D$. If $D$ is a regular Dirac operator for $\mathscr{B}$, then an automorphism of $S$ is automatically an automorphism of $\mathscr{B}$. If $U$ is a pre-spectral triple automorphism, then it defines by condition 3 an automorphism $\alpha_{U}$ of $\mathscr{A}$. Hence, we have a homomorphism $\alpha: U \mapsto \alpha_{U}$ from $\operatorname{Aut}(\mathscr{T})$ to $\operatorname{Aut}(\mathscr{A})$. We call $\operatorname{Vert}(\mathscr{T})$ the kernel of $\alpha$. Its elements will be called vertical automorphisms of $\mathscr{T}$. Correspondingly, we call $\operatorname{Hor}(\mathscr{T})$ the image of $\alpha$ and we call its elements the horizontal automorphisms. We thus have the exact sequence

$$
1 \longrightarrow \operatorname{Vert}(\mathscr{T}) \longrightarrow \operatorname{Aut}(\mathscr{T}) \stackrel{\alpha}{\longrightarrow} \operatorname{Hor}(\mathscr{T}) \longrightarrow 1
$$

If $\alpha$ has a section, i.e. a group homorphism $\beta$ such that $\alpha \circ \beta=\mathrm{Id}$, then $\operatorname{Aut}(\mathscr{T})$ will be the semidirect product $\operatorname{Vert}(\mathscr{T}) \ltimes \operatorname{Hor}(\mathscr{T})$, by the splitting lemma. Since algebraic backgrounds and spectral triples are pre-spectral triples, these considerations also apply to these structures. For example, at the end of section 3, we have attached a pre-spectral triple $\mathscr{T}_{M}$ to a parallelizable manifold $M$ of dimension $n$, and if we add the bimodule $\Omega^{1}$ of $\Gamma$-valued fields to it, we obtain an algebraic background $\mathscr{B}_{M}=\left(\mathscr{T}_{M}, \Omega^{1}\right)$. If we add the Dirac operator $D(e)$ to $\mathscr{T}_{M}$, we obtain a spectral triple $S_{M}(e)=\left(\mathscr{T}_{M}, D(e)\right)$. In all dimensions we have:

$$
\operatorname{Aut}\left(\mathscr{B}_{M}\right)=\operatorname{Diff}(M) \ltimes \Gamma(\operatorname{Spin}(s, t))
$$

and

$$
\operatorname{Aut}\left(S_{M}(e)\right)=\operatorname{Isom}\left(M, g_{e}\right) \ltimes \Gamma(\operatorname{Spin}(s, t))
$$

where $\operatorname{Isom}\left(M, g_{e}\right)$ is the isometry group of the metric $g_{e}$ defined by the tetrad $e$, and $\Gamma(\operatorname{Spin}(s, t))$ is the group of $\operatorname{Spin}(s, t)$-valued maps, i.e. LLT generalized to any signature. On the other hands, if $n \geq 6$, one has

$$
\operatorname{Aut}\left(\mathscr{T}_{M}\right) \supset \operatorname{Diff}(M) \ltimes \Gamma(\operatorname{Spin}(s, t))
$$

where the inclusion is strict. We see that from the point of view of the symmetry group, pre-spectral triples lack the clear geometric interpretation of both algebraic backgrounds and spectral triples. However we do have in all dimensions $\operatorname{Hor}\left(\mathscr{T}_{M}\right)=\operatorname{Hor}\left(\mathscr{B}_{M}\right)=\operatorname{Diff}(M)$.

Another important class of examples of pre-spectral triple automorphisms is given by gauge transformations. It is easy to check that $\Upsilon$ defined in (2.24) is a group homomorphism from the unitary group of the algebra to the group of vertical automorphisms of any almost-commutative pre-spectral triple. Under the first-order condition its image also lies in the group of algebraic background automorphisms. We see here that simple group-theoretic considerations can shed a new light on the definition of $\Upsilon$ and the first-order condition.

Let us now consider the question of compatible Dirac operators. It can be shown [16] that the configuration space of $\mathscr{B}_{M}$ is of the form

$$
\mathscr{D}\left(\mathscr{B}_{M}\right)=\Delta\left(\mathscr{B}_{M}\right) \oplus \mathscr{Z}\left(\mathscr{B}_{M}\right),
$$

where $\Delta\left(\mathscr{B}_{M}\right)$ is by definition the space of metric Dirac operators and $\mathscr{Z}\left(\mathscr{B}_{M}\right)$ is the space of centralizing fields. Metric Dirac operators ${ }^{9}$ are in bijective correspondance with tetrads on $M$, so

\footnotetext{
${ }^{9} \mathrm{~A}$ metric Dirac operator is the canonical Dirac operator associated to a tetrad $e^{\prime}$ rescaled in order to be self-adjoint for the Krein product (2.6), defined thanks to the origin tetrad $e$. For more details see [16].
} 
the space $\Delta\left(\mathscr{B}_{M}\right)$ exactly captures the degrees of freedom of tetradic gravity. A centralizing field is a multiplication operator of the form

$$
(\zeta \Psi)_{x}=\zeta_{x} \Psi_{x}
$$

where $\zeta_{x}$ is self-adjoint and commutes with the chirality and real structure. In the four-dimensional case, such an object is necessarily of the form $i \sum_{a<b<c} \gamma_{a} \gamma_{b} \gamma_{c} \zeta^{a b c}$, i.e. a 3-form field (or dually a pseudo-vector field). We see then that there are extra degrees of freedom with respect to tetradic GR, and we do not know whether they have a physical interpretation. A first impulse is to get rid of them, and this can be done using the fact that the two summands of (4.2) comprise very different objects: differential operators on one hand, and multiplication operators on the other (intuitively this is akin to the orthogonal decomposition of matrices into diagonal and off-diagonal ones). It follows that the projection operator on $\Delta\left(\mathscr{B}_{M}\right)$ with respect to $\mathscr{Z}\left(\mathscr{B}_{M}\right)$ is invariant under $\operatorname{Aut}\left(\mathscr{B}_{M}\right)$ and can be inserted into an action functional, effectively suppressing the centralizing fields.

We can define the configuration space of a pre-spectral triple in the same way as we did for algebraic backgrounds: an operator $D$ is in the configuration space of $\mathscr{T}$ iff $(\mathscr{T}, D)$ is a spectral triple. It can be shown ${ }^{10}$ that the configuration space of $\mathscr{T}_{M}$ is exactly the same as that of $\mathscr{B}_{M}$. Hence, we have $\mathscr{D}\left(\mathscr{T}_{M}\right)=\mathscr{D}\left(\mathscr{B}_{M}\right)$ and $\operatorname{Hor}\left(\mathscr{T}_{M}\right)=\operatorname{Hor}\left(\mathscr{B}_{M}\right)$, and it could be argued that the manifold case is not decisive enough to opt in favour of algebraic backgrounds rather than prespectral triples as a noncommutative framework for gravity theories. However we will see in the next section that finite models of manifolds make the difference between the two much more clear cut.

\section{Finite weighted graphs}

Let $G=(V, E, s, t)$ be a directed graph with vertex set $V$, edge set $E$, and source/target functions $s, t: E \rightarrow V$. Each positive "weight" function $w: E \rightarrow \mathbb{R}_{+}^{*}$ defines a geodesic distance $d_{w}$, and $\left(G, d_{w}\right)$ can be seen as a discrete manifold. We would like first to build a spectral triple on which Connes' distance formula (2.1) reproduces $d_{w}$. A naive attempt using the weighted adjacency matrix does not work and one is forced to introduce multiplicities [41]. A general solution is given by the split graph spectral triple [42], which we now describe. We first define the split graph $\tilde{E}:=E \times\{-,+\}$, which can be seen as the disconnected sum of the edges of $G$ (see figure 1).
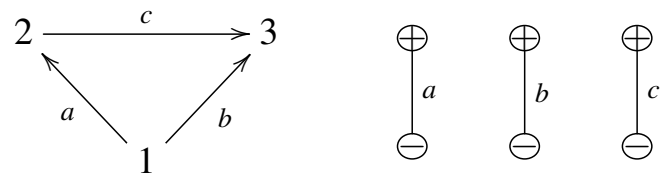

Figure 1: A directed graph and its split version

Then we let $\mathscr{A}=\mathbb{R}^{V}, \mathscr{H}=L^{2}(\tilde{E})=\mathbb{C}^{E} \otimes \mathbb{C}^{2}$, which we equip with the canonical scalar product. If we denote by $e^{-}$(resp. $e^{+}$) the source (resp. target) of the the edge $e$, then we can

\footnotetext{
${ }^{10}$ See the proof of theorem 11.2 in [40]. Note that in the case of algebraic backgrounds the proof is much more direct.
} 
define a projection map from $\tilde{E}$ to $V$ by $(e, \pm) \mapsto e^{ \pm}$. Using this notation, the action of a function $a \in \mathscr{A}$ which is defined on $V$ on an element of $\mathscr{H}$ which is a function on $\tilde{E}$ can be written as

$$
\pi(a) F(e, \pm):=a\left(e^{ \pm}\right) F(e, \pm)
$$

It will be useful to introduce yet another notation: for an edge $e$, we write $\mathscr{H}_{(e, \pm)} \subset \mathscr{H}$ for the subspace of functions vanishing outside $(e, \pm), \mathscr{H}_{e}:=\mathscr{H}_{(e,-)} \oplus \mathscr{H}_{(e,+)}$ and for a vertex $v$, we $\mathscr{H}_{v}:=\bigoplus_{(e, \pm) \mid e^{ \pm}=v} \mathscr{H}_{(e, \pm)}$. Using the identification $\mathscr{H}=\bigoplus_{e \in E} \mathscr{H}_{e} \simeq \bigoplus_{e \in E} \mathbb{C}^{2}$, we can write $\pi(a)$ in matrix form as

$$
\pi(a)=\bigoplus_{e}\left(\begin{array}{cc}
a\left(e^{-}\right) & 0 \\
0 & a\left(e^{+}\right)
\end{array}\right)
$$

whereas in the decomposition $\mathscr{H}=\bigoplus_{v \in V} \mathscr{H}_{v}$ we have

$$
\pi(a)=\bigoplus_{v} a(v) I_{d(v)},
$$

where $d(v)$ is the degree of the vertex $v$, and also the multiplicity of the evaluation at $v$ as a subrepresentation of $\pi$. With the same notation as in (5.2), we can define the chirality so that it is consistent with the orientation chosen on the graph by:

$$
\chi=\bigoplus_{e}\left(\begin{array}{cc}
-1 & 0 \\
0 & 1
\end{array}\right)
$$

Writing $\mathscr{H}^{+}$and $\mathscr{H}^{-}$the eigenspaces of $\chi$, we introduce for later use the subspaces $\mathscr{H}_{v}^{+}:=$ $\mathscr{H}_{v} \cap \mathscr{H}^{+}=\bigoplus_{(e,+) \mid e^{+}=v} \mathscr{H}_{(e,+)}$ and $\mathscr{H}_{v}^{-}:=\mathscr{H}_{v} \cap \mathscr{H}^{-}=\bigoplus_{(e,-) \mid e^{-}=v} \mathscr{H}_{(e,-)}$. The dimension of $\mathscr{H}_{v}^{+}$and $\mathscr{H}_{v}^{-}$are respectively the in-degree $d_{\text {in }}(v)$ and the out-degree $d_{\text {out }}(v)$, i.e. the number of incoming/outgoing edges at $v$.

The real structure $J$ is just complex conjugation in the canonical basis. Finally, the Dirac operator $D_{w}$ is

$$
D_{w}=\bigoplus_{e \in E} \frac{1}{w(e)}\left(\begin{array}{ll}
0 & 1 \\
1 & 0
\end{array}\right)
$$

One can prove [42] that $S(G, w):=\left(\mathscr{A}, \mathscr{H}, \pi, D_{w}, \chi, J\right)$ is a spectral triple of $\mathrm{KO}$ dimension 0 , satisfying the order 0 but, interestingly, not the order 1 condition. Moreover, the distance defined by (2.1) coincides with the geodesic distance defined by $w$. It is also easy to see that the bimodule of 1-forms $\Omega^{1}$ defined by $D_{w}$ is independent of $w$ and contains the operators of the form

$$
\omega=\bigoplus_{e \in E}\left(\begin{array}{cc}
0 & \omega_{e}^{+} \\
\omega_{e}^{-} & 0
\end{array}\right) \text {, with } \omega_{e}^{ \pm} \in \mathbb{R} .
$$

Remark: Note that the map $a \mapsto[D, \pi(a)]$ has values in anti-selfadjoint 1-forms, and since (5.5) is antiselfadjoint iff $\omega_{e}^{+}$and $\omega_{e}^{-}$are opposite, hence when $\omega$ depends bijectively on a function defined on $E$, this is consistent with the theorem proved in [43] that FODC on countable discrete sets are in bijective correspondence with digraph structures, the 1-forms being generated by the edges.

Now we change our viewpoint and consider the background $\mathscr{B}(G):=\left(\mathscr{A}, \mathscr{H}, \pi, \Omega^{1}, \chi, J\right)$. Let us look for the automorphisms of $\mathscr{B}(G)$. Recall that a permutation $\sigma$ of the vertices is called a graph automorphism iff $\sigma$ respects the edges, i.e. if $v$ and $v^{\prime}$ are connected by an edge, then so are 
$\sigma(v)$ and $\sigma\left(v^{\prime}\right)$. It follows that $\sigma$ acts on the set of edges. We moreover say that $\sigma$ is oriented if it respects the orientation. We write $\operatorname{Aut}_{\text {or }}(G)$ for the group of oriented graph automorphisms of $G$. Given an element $\sigma$ of this group, one defines $U_{\sigma}$ by

$$
U_{\sigma} F(e, \pm)=F\left(\sigma^{-1}(e), \pm\right)
$$

This is easy to show that $\beta: \sigma \mapsto U_{\sigma}$ is a group homomorphism from $\operatorname{Aut}_{\text {or }}(G)$ to $\operatorname{Aut}(\mathscr{B}(G))$.

Now let $U \in \operatorname{Aut}(\mathscr{B}(G))$. Since $\operatorname{Aut}(\mathscr{A})$ is the permutation group of $V$, acting by pull-back, there is a permutation $\sigma$ of $V$ such that $\alpha_{U}=\sigma_{*}$, or in other words:

$$
\forall a \in \mathscr{A}, U \pi(a) U^{-1}=\pi\left(a \circ \sigma^{-1}\right) .
$$

Using for $a$ the Dirac $\delta$-function at $v \in V$, (5.7) shows that $U \mathscr{H}_{v}=\mathscr{H}_{\sigma(v)}$. Now let us use $U \Omega^{1} U^{-1}=\Omega^{1}$. Let $e \in E$ and $\omega$ be the 1-form defined by $\omega F(e, \pm)=F(e, \mp)$ and $\omega F\left(e^{\prime}, \pm\right)=0$ for $e^{\prime} \neq e$. Then $\omega$ is a 1-form which has rank 2, is selfadjoint, and whose square is the orthogonal projector on $\mathscr{H}_{e}$. It follows that $\omega^{\prime}:=U \omega U^{-1}$ is a 1-form which has rank two, is selfadjoint, and whose square is the orthogonal projector on $U \mathscr{H}_{e}$. Now a rank 2 selfadjoint 1-form is necessarily of the form $\omega^{\prime} F\left(e^{\prime}, \pm\right)=t F\left(e^{\prime}, \mp\right), \omega^{\prime} F\left(e^{\prime \prime}, \pm\right)=0$ for $e^{\prime \prime} \neq e^{\prime}$ and the condition on the square shows that $t=1$. We conclude that $U \mathscr{H}_{e}=\mathscr{H}_{e^{\prime}}$. Now $\mathscr{H}_{e}=\mathscr{H}_{(e,-)} \oplus \mathscr{H}_{(e,+)}, \mathscr{H}_{e^{\prime}}=\mathscr{H}_{\left(e^{\prime},-\right)} \oplus \mathscr{H}_{\left(e^{\prime},+\right)}$, and $U \mathscr{H}_{e^{ \pm}}=\mathscr{H}_{\sigma\left(e^{ \pm}\right)}$. This shows that $\sigma\left(e^{+}\right)$and $\sigma\left(e^{-}\right)$belong to the same edge $e^{\prime}$, hence that $\sigma$ is a graph automorphism, sending $e$ to $e^{\prime}$. Now if $z \in \mathscr{H}_{e^{+}}$, then $U \chi z=U z$, and $U \chi z=\chi U z$ show that $\chi(U z)=U z$, hence $U$ sends $\mathscr{H}_{(e,+)}$ to $\mathscr{H}_{(\sigma(e),+)}$, which shows that $\sigma$ is an oriented graph automorphism. Hence we have proven that $\operatorname{Hor}(\mathscr{B}(G)) \simeq \operatorname{Aut}_{\text {or }}(G)$, and since $\beta$ is immediately seen to be a section of $\alpha$, we conclude that $\operatorname{Aut}(\mathscr{B}(G))$ is the semi-direct product of the horizontal and vertical automorphism groups. There remains to identify the latter one. For this, consider a background automorphism $U$ which commutes with the elements of $\pi(\mathscr{A})$. Then the above proof shows that it sends $\mathscr{H}_{\left(e^{ \pm}\right)}$to itself for every $e$. Since it is unitary and commutes with complex conjugation, it is of the form

$$
U F(e, \pm)=s_{e}^{ \pm} F(e, \pm)
$$

where $s_{e}^{ \pm}$is a sign. We have thus proven that:

$$
\operatorname{Aut}(\mathscr{B}(G))) \simeq \operatorname{Aut}_{\mathrm{or}}(G) \ltimes\{-1 ; 1\}^{\tilde{E}}
$$

Let us now consider an element $U$ of $\operatorname{Aut}(S(G, w))$. Since the vertical elements (5.8) do not commute with $D_{w}$ unless they are trivial, we see by (5.9) that $U$ is of the form (5.6), and a quick calculation shows that $U_{\sigma}$ commutes with $D_{w}$ iff $\sigma$ preserves $w$. Thus we have

$$
\operatorname{Aut}(S(G, w)) \simeq \operatorname{Aut}_{\mathrm{or}}(G, w)
$$

Remark: The preservation of $w$ implies that of $d_{w}$. If $w$ satisfies the triangle inequality, then preserving $w$ or $d_{w}$ is equivalent. It this case $\operatorname{Aut}(S(G, w))$ is isomorphic to the group of oriented $d_{w}$-isometries of $G$. For an example of a graph automorphism preserving $d_{w}$ but not $w$, consider the vertical symmetry of the graph displayed in figure 2.

Now let us consider the pre-spectral triple $\mathscr{T}(G)=(\mathscr{A}, \mathscr{H}, \pi, \chi, J)$. Let $U$ be an element of $\operatorname{Aut}(\mathscr{T}(G))$ and $\sigma=\alpha(U)$. As in the above proof, we see using $U \mathscr{H}_{v}=\mathscr{H}_{\sigma(v)}$ and $U \chi=\chi U$ 


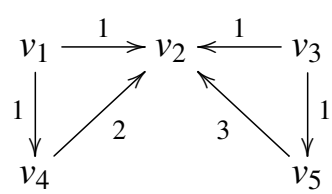

Figure 2: The vertical symmetry of this directed graph does not conserve the weight function $w$, but does respect the geodesic distance $d_{w}$.

that $U \mathscr{H}_{v}^{ \pm}=\mathscr{H}_{\sigma(v)}^{ \pm}$. It follows that $\sigma$ is a permutation of the vertices which respects the in and out degrees. Conversely, let $\mathfrak{S}\left(V ; d_{\text {in }}, d_{\text {out }}\right)$ be the group of such permutations. Let us construct a section $\beta$ of $\alpha$. For each $v$ we have an identification $\mathscr{H}_{v}^{-}=\mathbb{C}^{d_{\text {out }}(v)}$ given by choosing the canonical basis elements (Dirac delta functions) corresponding to the edges $e$ such that $e^{-}=v$ ordered in a particular way. By a similar choice of basis, we fix identifications $\mathscr{H}_{v}^{+} \simeq \mathbb{C}^{d_{\text {in }}(v)}$ one and for all. Let $V=\bigcup_{i=1, \ldots, k} V_{i}$ be the partition of $V$ into classes of vertices of the same in and out degrees. Then for every $\sigma \in \mathfrak{S}_{+}$and $i \in\{1, \ldots, k\}, \sigma$ induces a permutation $\sigma_{i}$ of the class $V_{i}$. We define the unitary operator $\beta(\sigma)$ by giving its matrix $M_{\sigma}$ in the block decomposition given by writing

$$
\mathscr{H}=\bigoplus_{i=1, \ldots, k} \mathscr{H}_{i}^{+} \oplus \mathscr{H}_{i}^{-}
$$

where

$$
\mathscr{H}_{i}^{ \pm}=\bigoplus_{v \in V_{i}} \mathscr{H}_{v}^{ \pm}
$$

Then

$$
M_{\sigma}=\bigoplus_{i=1, \ldots, k} P_{\sigma_{i}}^{+} \oplus P_{\sigma_{i}}^{-}
$$

where $P_{\sigma_{i}}^{ \pm}$is the block-permutation matrix corresponding to the action of $\sigma_{i}$ on the summands of (5.12). Then $\beta(\sigma)$ is unitary and commutes with $\chi$ and $J$ by construction. If we now consider the unitary operators commuting with $\pi(\mathscr{A}), \chi$ and $J$, we see easily that they are of the form

$$
O=\bigoplus_{i=1, \ldots, k} O_{i}^{+} \oplus O_{i}^{-}
$$

where $O_{i}^{ \pm}$is in the orthogonal group $O\left(d_{\text {out } / \text { in }}\left(v_{i}\right)\right)$, according to the case. Hence we see that the horizontal part of $\operatorname{Aut}(\mathscr{T}(G))$ is $\mathfrak{S}\left(V ; d_{\text {in }}, d_{\text {out }}\right)$, while the vertical part is the direct product $\prod_{i=1, \ldots, k} O\left(d_{\text {in }}\left(v_{i}\right)\right) \times O\left(d_{\text {out }}\left(v_{i}\right)\right)$.

Let us illustrate these general considerations with the "pyramid graph" of figure 3. In this case, the vertices fall into two classes: $V_{1}=\{5\}$ and $V_{2}=V \backslash V_{1}$. We also have:

$$
\begin{aligned}
& \mathscr{H}_{1}^{+}=\mathscr{H}_{v_{5}}^{+}=\bigoplus_{i=1, \ldots, 4} \mathscr{H}_{\left(f_{i},+\right)}, \quad \mathscr{H}_{1}^{-}=\{0\}, \\
& \mathscr{H}_{2}^{+}=\bigoplus_{i=1, \ldots, 4} \mathscr{H}_{v_{i}}^{+}, \quad \mathscr{H}_{2}^{-}=\bigoplus_{i=1, \ldots, 4} \mathscr{H}_{v_{i}}^{-},
\end{aligned}
$$

with

$$
\mathscr{H}_{v_{1}}^{+}=\mathscr{H}_{\left(e_{4},+\right)}, \mathscr{H}_{v_{2}}^{+}=\mathscr{H}_{\left(e_{1},+\right)}, \mathscr{H}_{v_{3}}^{+}=\mathscr{H}_{\left(e_{2},+\right)}, \mathscr{H}_{v_{4}}^{+}=\mathscr{H}_{\left(e_{3},+\right)},
$$




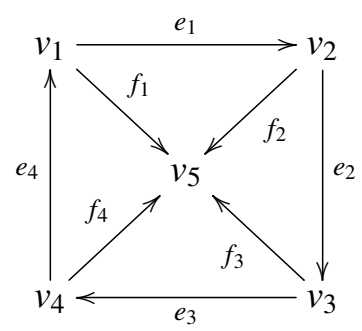

Figure 3: A directed pyramid.

and for all $i=1, \ldots, 4$

$$
\mathscr{H}_{v_{i}}^{-}=\mathscr{H}_{\left(e_{i},-\right)} \oplus \mathscr{H}_{\left(f_{i},-\right)} .
$$

Moreover, by choosing the delta function on each split graph element as a basis, we obtain the identifications $\mathscr{H}_{v_{i}}^{+} \simeq \mathbb{C}$ and $\mathscr{H}_{v_{i}}^{-} \simeq \mathbb{C}^{2}$ for each $i=1, \ldots, 4$. Let us now consider the permutation $\sigma$ sending $v_{1}, \ldots, v_{5}$ to $v_{3}, v_{1}, v_{4}, v_{2}, v_{5}$ in that order. Note that $\sigma$ respects the in/out-degrees while not being a graph automorphism, since it sends $v_{1}, v_{2}$, which are connected by $e_{1}$, to $v_{1}, v_{3}$, which are not connected by an edge. Using (5.13) we obtain the operator $\beta(\sigma)$ whose matrix in the chosen basis is

$$
M_{\sigma}=1 \oplus\left(\begin{array}{cccc}
0 & 1 & 0 & 0 \\
0 & 0 & 0 & 1 \\
1 & 0 & 0 & 0 \\
0 & 0 & 1 & 0
\end{array}\right) \oplus\left(\begin{array}{cccc}
0 & 1_{2} & 0 & 0 \\
0 & 0 & 0 & 1_{2} \\
1_{2} & 0 & 0 & 0 \\
0 & 0 & 1_{2} & 0
\end{array}\right)
$$

Let us now return to the general case and look for the compatible Dirac operators on the background $\mathscr{B}(G)$. For such an operator $D$ and any $v \in V$ there exists an anti-selfadjoint $\omega_{v} \in \Omega^{1}$ such that $\left[D, \pi\left(\delta_{v}\right)\right]=\omega_{x}$, i.e. there exists a real function $f_{v}$ which is defined on $E$, and is such that for all $(e, \pm) \in \tilde{E}$,

$$
\left[D, \delta_{v}\right] F(e, \pm)= \pm f_{v}(e) F(e, \mp)
$$

where we have identified the Dirac delta function at $v$ and its image under $\pi$. Let us now make the following two observations:

1. From $\delta_{v}^{2}=\delta_{v}$ and the Leibniz rule for $[D,$.$] we obtain for any F \in \mathscr{H}$

$$
\begin{aligned}
{\left[D, \delta_{v}\right] F(e,+) } & =\delta_{v}\left[D, \delta_{v}\right] F(e,+)+\left[D, \delta_{v}\right] \delta_{v} F(e,+) \\
\Rightarrow f_{v}(e) F(e,-) & =\delta_{v}\left(e^{+}\right) f_{v}(e) F(e,-)+f_{v}(e) \delta_{v}\left(e^{-}\right) F(e,-)
\end{aligned}
$$

which yields

$$
f_{v}(e)=0 \text { if } v \notin e
$$

2. From $\delta_{e^{+}} \delta_{e^{-}}=0$ and the Leibniz rule again we obtain for all $F$ :

$$
\begin{aligned}
{\left[D, \delta_{e^{+}}\right] \delta_{e^{-}} F(e,+)+\delta_{e^{+}}\left[D, \delta_{e^{-}}\right] F(e,+) } & =0 \\
\Rightarrow f_{e^{+}}(e)\left(\delta_{e^{-}} F\right)(e,-)+\delta_{e^{+}}\left(e^{+}\right) f_{e^{-}}(e) F(e,-) & =0 \\
\Rightarrow f_{e^{+}}(e)+f_{e^{-}}(e) & =0
\end{aligned}
$$


Let us call $D^{0}$ the operator defined by

$$
\left(D^{0} F\right)(e, \pm)= \pm f_{e^{\mp}}(e) F(e, \mp)=f_{e^{-}}(e) F(e, \mp)
$$

for all $e \in E$. Then one has

$$
\begin{aligned}
& {\left[D^{0}, \delta_{v}\right] F(e,+)=f_{e^{-}}(e)\left(\delta_{v}\left(e^{-}\right)-\delta_{v}\left(e^{+}\right)\right) F(e,-)} \\
& {\left[D^{0}, \delta_{v}\right] F(e,-)=f_{e^{-}}(e)\left(\delta_{v}\left(e^{+}\right)-\delta_{v}\left(e^{-}\right)\right) F(e,+)}
\end{aligned}
$$

It is easy to show that $\left[D^{0}, \delta_{v}\right]=\left[D, \delta_{v}\right]$ for every vertex $v$ using (5.19), (5.21) and (5.22). Thus $\zeta:=$ $D-D^{0}$ commutes with $\pi(\mathscr{A})$ by linearity. By (5.3) we have $\zeta=\bigoplus_{v \in V} \zeta_{v}$, where $\zeta_{v} \in \operatorname{End}\left(\mathscr{H}_{v}\right)$ is selfadjoint, commutes with $J$ and anticommutes with $\chi$. It is thus characterized by a "transfert matrix" $t_{v} \in \mathscr{M}_{d_{\text {in }}(v), d_{\text {out }}(v)}(\mathbb{R})$, while $D^{0}$ is characterized by the function $e \mapsto f_{e^{-}}(e)$, which plays the role of the inverse weight $1 / w$. Thus we have proven that the configuration space of $\mathscr{B}(G)$ is the direct $\operatorname{sum} \Delta \oplus \mathscr{Z}$, where

$$
\Delta=\mathscr{C}(E, \mathbb{R})
$$

is the space of inverse weights ${ }^{11}$ and

$$
\mathscr{Z} \simeq \bigoplus_{v \in V} \mathscr{M}_{d_{\text {in }}(v), d_{\text {out }}(v)}(\mathbb{R})
$$

is the space of transfert matrices. We note that $\Delta$ and $\mathscr{Z}$ are orthogonal with respect to the HilbertSchimdt scalar product. Note also that if we remove the compatibility condition with $\Omega^{1}$, we obtain a much larger configuration space, whose elements can mix subpaces $\mathscr{H}_{v}$ for non-connected vertices, and do not have a simple interpretation.

In summary, we have obtained that:

- $\operatorname{Hor}(\mathscr{T}(G))=\mathfrak{S}\left(V ; d_{\text {in }}, d_{\text {out }}\right)$,

- $\operatorname{Hor}(\mathscr{B}(G))=\operatorname{Aut}_{\text {or }}(G)$,

- $\operatorname{Hor}(S(G))=\operatorname{Aut}_{\text {or }}(G, w)$.

Moreover the non-centralizing compatible Dirac operators on $\mathscr{B}(G)$ bijectively corresponds to generalized weights on the edges of the graph, while the corresponding objects on $\mathscr{T}(G)$ are much more general. We believe that this would suffice to justify the use of algebraic backgrounds for finite gravity models.

\section{The algebraic background of the Lorentzian Standard Model}

We now turn our attention to a construction of immediate relevance for particle physics. We consider the finite background $\mathscr{B}_{F}=\left(\mathscr{A}_{F}, \mathscr{H}_{F}, \pi_{F}, \chi_{F}, J_{F}, \Omega_{F}^{1}\right)$, where $\mathscr{A}_{F}, \mathscr{H}_{F}, \pi_{F}, \chi_{F}$ and $J_{F}$ have already been defined in equations (2.10) to (2.14). The Krein product on $\mathscr{H}_{F}$ is

$$
\left(\psi, \psi^{\prime}\right)_{F}=\left\langle\psi, \chi_{F} \psi^{\prime}\right\rangle
$$

\footnotetext{
${ }^{11}$ Note that the elements of $\Delta$ can take negative values, but this does not change Connes' distance which only depends on the absolute value of the weight function.
} 
where $\langle.,$.$\rangle is the canonical scalar product on \mathscr{H}_{F}$. The chirality is introduced in order to compensate for the $\beta$ factor coming from formula (2.9): this is where the non-Euclidean character of the base manifold is transmitted to the finite part. Another way to look at this phenomenon is to write down the Fermionic Lagrangian and observe that $\chi_{F}$ is needed in order to obtain all the correct signs [15]. Finally we need to define the bimodule $\Omega_{F}^{1}$. It is defined to contain the matrices of the form

$$
\omega_{F}=\left(\begin{array}{cccc}
0 & Y_{0}^{\dagger} \tilde{q}_{1} & 0 & 0 \\
\tilde{q}_{2} Y_{0} & 0 & 0 & 0 \\
0 & 0 & 0 & 0 \\
0 & 0 & 0 & 0
\end{array}\right), q_{1}, q_{2} \in \mathbb{H}
$$

where we have used the notation $\tilde{q}:=q \otimes 1_{4} \otimes 1_{3}$. To motivate the definition of $\Omega_{F}^{1}$ we can apply the first and second order condition $[9,10]$ or the first-order condition and the massless photon condition [23], as we did for $D_{F}$.

The background of the Lorentzian ${ }^{12}$ Standard Model is thus $\mathscr{B}_{\mathrm{SM}}=\mathscr{B}_{M} \hat{\otimes} \mathscr{B}_{F}$ where $M$ is a four-dimensional Lorentzian spin manifold of one's choice. We can now compute the automorphism group of this manifold and we find the following result [44].

Theorem 1. If $\operatorname{Hom}\left(\pi_{1}(M), \mathbb{Z}_{6}\right)=\{0\}$ and if $Y_{0}$ is generic (see the remark below), then $\operatorname{Aut}\left(\mathscr{B}_{M} \hat{\otimes} \mathscr{B}_{F}\right)$ is generated by the elements of the form:

1. $U_{\theta} \hat{\otimes} 1$, where $\theta \in \operatorname{Diff}(M)$, as defined in (3.4),

2. $U_{\Sigma} \hat{\otimes} 1$, where $\Sigma$ is a LLT, as defined in (3.5),

3. local gauge transformations $1 \hat{\otimes} \Upsilon(u)$ where $u \in \mathscr{C}^{\infty}\left(M, U\left(\mathscr{A}_{F}\right)\right)$, and $\Upsilon$ is defined in (2.24),

4. local $B-L$ transformations $1 \hat{\otimes} g_{B-L}(\varphi)$, where $\varphi \in \mathscr{C}^{\infty}(M, \mathbb{R})$, and

$$
g_{B-L}(t)=\operatorname{diag}\left(e^{-i t} 1_{2} \oplus e^{i t / 3} 1_{2} \otimes 1_{3}, e^{-i t} 1_{2} \oplus e^{i t / 3} 1_{2} \otimes 1_{3}, c, c\right) \otimes 1_{3}
$$

Remark: The genericity hypothesis appearing in the theorem is the following: we say that $Y_{0}=\left(\begin{array}{cc}Y_{V} & 0 \\ 0 & Y_{e}\end{array}\right) \oplus$ $\left(\begin{array}{cc}1_{3} \otimes Y_{u} & 0 \\ 0 & 1_{3} \otimes Y_{d}\end{array}\right)$ is generic if it is invertible and every matrix commuting with $Y_{v} Y_{v}^{\dagger}$ and $Y_{e} Y_{e}^{\dagger}$ (resp. with $Y_{u} Y_{u}^{\dagger}$ and $\left.Y_{d} Y_{d}^{\dagger}\right)$ is a scalar. Note that this hypothesis is not verified when one right-handed neutrino remains massless (see [45] for a review of such models). Note also that without the topological hypothesis on $M$ there could exist mixes between LLT and finite vertical automorphisms which could not be disentangled globally [44].

The real surprise here comes from the fourth kind of automorphisms. It is a fact that the Lagrangian of the Standard Model conserves both baryon and lepton numbers. It has been hypothesized long ago [46] that the B-L combination of these global "accidental" symmetries could be gauged giving rise to the only abelian extension of the Standard Model which is anomaly free without adding new Fermions. We see that in the context of algebraic backgrounds we do not have any choice: the gauged $B-L$ symmetry is part of the automorphism group, on the same footing

\footnotetext{
${ }^{12}$ Or rather anti-Lorentzian since we work with the mostly minus signature.
} 
as the other transformations. Hence we must include the associated gauge fields in order to maintain automorphism invariance (which is here a noncommutative version of GR's diffeomorphism invariance). In particular we see that the algebraic background framework is even more restrictive than the spectral triple framework, since only the $U(1)_{\mathrm{B}-\mathrm{L}}$-extended version of the Standard Model can be consistently formulated within it. It is a fortunate circumstance that this extension is one of the best motivated for a number of both phenomenological and theoretical considerations $[47,48,49,50]$.

As for the configuration space of $\mathscr{B}:=\mathscr{B}_{M} \hat{\otimes}_{\mathscr{B}_{F}}$, it is found to be

$$
\mathscr{D}_{\mathscr{B}}=\mathscr{D}_{\text {Gravity }} \oplus \mathscr{D}_{\Omega^{1}} \oplus \mathscr{D}_{B-L} \oplus \mathscr{D}_{\sigma} \oplus \mathscr{D}_{\text {flavour }} .
$$

The gravity part is $\Delta\left(\mathscr{B}_{M}\right) \hat{\otimes} 1$ (see (4.2), while $\mathscr{D}_{\Omega^{1}}$ contains the usual gauge and Higgs fields of the Standard Model, $\mathscr{D}_{B-L}$ contains the $Z_{B-L}^{\prime}$ boson, and $\mathscr{D}_{\sigma}$ a new complex scalar which breaks the $U(1)_{B-L}$ symmetry and is of $B-L$ charge 2 . Finally $\mathscr{D}_{\text {flavour }}$ contains operators which only act on flavour space. They can be suppressed by a gauge-invariant projection [44], and there remains a model with configuration space

$$
\mathscr{D}_{\mathscr{B}}^{\min }=\mathscr{D}_{\text {Gravity }} \oplus \mathscr{D}_{\Omega^{1}} \oplus \mathscr{D}_{B-L} \oplus \mathscr{D}_{\sigma}
$$

where the exponent "min" means that it is the smallest subspace of the configuration space which is invariant by the symmetry group and contains a field giving rise to neutrino masses.

\section{Conclusion, outlook}

We see that in the framework of algebraic backgrounds the scalar field which was needed to push down the prediction of the Higgs mass appears without breaking the order 1 condition but rather by extending the bosonic configuration space from the space of fluctuations to the space of all compatible Dirac operators, in a move which would be similar to embedding gauge theory into a Kaluza-Klein model. The spectral action applied to the configuration space (6.4) in Euclidean signature, followed by a Wick rotation, would thus yield predictions fully consistent with current observations. Unfortunately, the only action principle in Noncommutative Geometry which is known to be compatible with Lorentzian signature [15] is the Connes-Lott one, but it is only defined on fluctuations. It is however possible to extend the finite background $\mathscr{B}_{M}$ in such a way that the space of fluctuated Dirac of the new total background is exactly the non-gravitational part of (6.4)! The extended algebra is $\mathscr{A}_{F}^{\text {ext }}:=\mathscr{A}_{F} \oplus \mathbb{C}$, represented on $\mathscr{K}_{F}$ by

$$
\pi_{F}^{\mathrm{ext}}(\lambda, q, m, \mu)=\operatorname{diag}\left(\tilde{q}_{\lambda}, \tilde{q}, \mu \otimes 1_{3} \oplus 1_{2} \otimes m \otimes 1_{3}, \mu \otimes 1_{3} \oplus 1_{2} \otimes m \otimes 1_{3}\right)
$$

The bimodule of extended 1-forms $\left(\Omega_{F}^{1}\right)^{\text {ext }}$ is defined by the requirement that $D_{F}$ still be a regular Dirac. A quick computation shows that it contains the matrices of the form

$$
\omega_{F}^{\mathrm{ext}}=\left(\begin{array}{cccc}
0 & Y_{0}^{\dagger} \tilde{q}_{1} & z_{2} M_{0}^{\dagger} & 0 \\
\tilde{q}_{2} Y_{0} & 0 & 0 & 0 \\
z_{1} M_{0} & 0 & 0 & 0 \\
0 & 0 & 0 & 0
\end{array}\right)
$$


with $q_{1}, q_{2} \in \mathbb{H}$ and $z_{1}, z_{2} \in \mathbb{C}$. Note that $\mathscr{B}_{F}^{\text {ext }}$ does not satisfy the order 1 condition. In this way this model is similar to those found in the approaches which make the $\sigma$-field appear by relaxing this condition $[6,7,8]$. In fact, it is immediately seen to be a submodel of Pati-Salam, but the fluctuations of the Dirac operator can be treated as usual, in contrast to [8]. This can be done thanks to a weak form of the order 1 condition, which still applies. The Connes-Lott action has been evaluated [28], and the renormalization group analysis will appear in the near future.

\section{References}

[1] A. Chamseddine and W. van Suijlekom, "A survey of spectral models of gravity coupled to matter." 2019.

[2] A. Connes and J. Lott, Particles models and noncommutative geometry, Nucl. Phys. Proc. Suppl. 18B (1991) 29.

[3] A. H. Chamseddine and A. Connes, Universal formula for noncommutative geometry actions: Unifications of gravity and the standard model, Phys. Rev. Lett. 77 (1996) 4868.

[4] A. Chamseddine, A. Connes and M. Marcolli, Gravity and the Standard Model with neutrino mixing, Adv. Theor. Math. Phys. 11 (2007) 991.

[5] A. Chamseddine and A. Connes, Resilience of the spectral standard model, JHEP 104 (2012).

[6] L. Boyle and S. Farnsworth, Rethinking Connes' approach to the standard model of partic le physics via non-commutative geometry, New J. Phys. 17 (2015) .

[7] A. Devastato, F. Lizzi and P. Martinetti, Higgs mass in noncommutative geometry, Fortsch. Phys. 62 (2014) .

[8] A. Chamseddine, A. Connes and W. van Suijlekom, Beyond the spectral standard model: emergence of Pati-Salam unification, J. High Energ. Phys. 2013: 132 (2013) .

[9] L. Boyle and S. Farnsworth, Non-commutative geometry, non-associative geometry and the Standard Model of particle physics, New J. Phys. 1616 (2014).

[10] C. Brouder, N. Bizi and F. Besnard, "The Standard Model as an extension of the noncommutative algebra of forms." 2015.

[11] L. Dabrowski, F. D'Andrea and A. Sitarz, The standard model in noncommutative geometry: fundamental fermions as internal forms, Lett. Math. Phys. 108 (2018) .

[12] L. Dabrowski and A. Sitarz, Fermion masses, mass-mixing and the almost commutative geometry of the standard model, J. High Energ. Phys. 68 (2019).

[13] A. Connes, On the spectral characterization of manifolds, J. Noncommut. Geom. 7 (2013) 1.

[14] K. van den Dungen, Krein spectral triples and the Fermionic action, Math. Phys. Anal. Geom. 19 (2016) .

[15] N. Bizi, Semi-Riemannian Noncommutative Geometry, Gauge Theory, and the Standard Model of Particle Physics, Ph.D. thesis, UPMC, Sorbonne Universités, 2018.

[16] F. Besnard, Algebraic backgrounds for noncommutative kaluza-klein theory. i. motivations and general framework, J. Math. Phys. 60 (2019) 123506.

[17] F. Besnard and N. Bizi, Doppler shift in semi-Riemannian signature and the non-uniqueness of the Krein space of spinors, J. Math. Phys. 60 (2019) . 
[18] C. Brouder, N. Bizi and F. Besnard, Space and time dimensions of algebras with application to Lorentzian noncommutative geometry and quantum electrodynamics, Journal of Mathematical Physics 59 (2018) .

[19] S. L. Woronowicz, Differential calculus on compact matrix pseudogroups (quantum groups), Commun. Math. Phys. 122 (1989) 125.

[20] A. Connes, Noncommutative Geometry. Academic Press, 1994.

[21] E. Minguzzi, Causality theory for closed cone structures with applications, Rev. Math. Phys. 31 (2019) .

[22] N. Franco, The lorentzian distance formula in noncommutativegeometry, in Non-Regular spacetime geometry, vol. 968, IOP Publishing, 2018.

[23] A. Connes and M. Marcolli, Noncommutative Geometry, Quantum Fields and Motives. AMS, 2008.

[24] F. Lizzi, G. Mangano, G. Miele and G. Sparano, Fermion Hilbert space and fermion doubling in the noncommutative geometry approach to gauge theories, Phys. Rev. D 55 (1997) 6357.

[25] J. W. Barrett, A Lorentzian version of the non-commutative geometry of the standard model of particle, J. Math. Phys. 48 (2007) 012303.

[26] F. Besnard, "On the uniqueness of Barrett's solution to the fermion doubling problem in noncommutative geometry." 2019.

[27] A. Connes, Noncommutative geometry and the standard model with neutrino mixing, JHEP 11 (2006)

[28] F. Besnard, “A $u(1)_{B-L}$ extension from noncommutative geometry.” 2019.

[29] A. H. Chamseddine and A. Connes, The spectral action principle, Commun. Math. Phys. 186 (1997) 731.

[30] W. D. van Suijlekom, Noncommutative Geometry and Particle Physics. Springer, 2015.

[31] C. Martín, J. Gracia-Bondía and J. Várilly, The Standard Model as noncommutative geometry: the low-energy regime, Phys. Repts 294 (1998).

[32] A. Connes and J. Lott, The metric aspect of noncommutative geometry, in New Symmetry Principles in Quantum Field Theory, J. F. et al, ed., vol. 295 of NATO ASI Series (Series B: Physics), pp. 53-93, Springer, Boston, MA, 1992.

[33] B. Iochum, D. Kastler and T. Schücker, Fuzzy mass relations for the higgs, Journal of Mathematical Physics 36 (1995) 6232.

[34] B. Iochum, D. Kastler and T. Schücker, "Fuzzy mass relations in the Standard Model.".

[35] M. Paschke, Mass relations in noncommutative geometry revisited, Phys. Lett. B 414 (1997) 323.

[36] H. Karimi, Implications of the complex singlet field for noncommutative geometry model, Journal of High Energy Physics 2017 (2017) 40.

[37] C. Rovelli, Spectral noncommutative geometry and quantization, Phys. Rev. Lett. 83 (1999) 1079.

[38] F. Besnard, Canonical quantization and the spectral action; a nice example, Journal of Geometry and Physics 57 (2007) 1757.

[39] C. Rovelli, "Lorentzian Connes distance, spectral graph distance and loop gravity.” 2014. 
[40] J. Gracia-Bondía, J. Várilly and H. Figueroa, Elements of Noncommutative Geometry. Birkhäuser, 2001.

[41] B. Iochum, T. Krajewski and P. Martinetti, Distances in finite spaces from noncommutative geometry, J. Geom. Phys. 37 (2001) 100.

[42] F. Besnard, "On the definition of spacetimes in noncommutative geometry: part ii." 2016.

[43] A. Dimakis and F. Müller-Hoissen, Discrete differential calculus, graphs, topologies and gauge theory, J. Math. Phys. 35 (1994) 6703.

[44] F. Besnard, Algebraic backgrounds for noncommutative kaluza-klein theory. ii. the almost-commutative case and the standard model, J. Math. Phys. 60 (2019) 123507.

[45] W.-L. Guo, Z.-Z. Xing and S. Zhou, Neutrino masses, lepton flavor mixing and leptogenesis in the minimal seesaw model, International Journal of Modern Physics E 16 (2007) 1.

[46] B. Holdom, Two u(1)'s and $\varepsilon$ charge shift, Phys. Lett. B 166 (1986) .

[47] T. Appelquist, B. Dobrescu and A. R. Hopper, Nonexotic neutral gauge bosons, Phys. Rev. D 68 (2002) .

[48] L. Basso, A. Belyaev, S. Moretti and C. H. Shepherd-Themistocleous, Phenomenology of the minimal $b-l$ extension of the standard model: $z^{\prime}$ and neutrinos, Phys. Rev. D 80 (2009) 055030.

[49] S. Iso, N. Okada and Y. Orikasa, Classically conformal b-l extended Standard Model, Phys. Lett. B 676 (2009) 81.

[50] L.Boyle, S. Farnsworth, J. Fitzgerald and M. Schade, "The Minimal Dimensionless Standard Model (MDSM) and its cosmology." 2011. 